\title{
PROTOCOLO DE TRATAMIENTO PSICÓLOGICO GRUPAL PARA ABORDAR SINTOMATOLOGÍA DE ANSIEDAD
}

\author{
M. CARMEN BENEDITO, M. CARMEN CARRIÓ, \\ GEMA DEL VALLE y AGUSTÍN DOMINGO
}

Unidad de Salud Mental, Centro de Especialidades de Burjassot, Área de Salud 05, Diputación de Valencia

(Aceptado en marzo de 2004)

\begin{abstract}
El presente trabajo propone un protocolo de terapia grupal para el tratamiento de los síntomas en lugar de los trastornos como estrategia para reducir la presión asistencial existente en las Unidades de Salud Mental. En concreto, se ofrece un protocolo para tratar sintomatología de ansiedad a nivel grupal desde la perspectiva cognitivo-conductual. Se incluye la descripción del contenido de cada sesión, un modelo explicativo de la ansiedad y un sistema de registro que contempla la psicopatología y las estrategias de afrontamiento de la ansiedad.

Palabras clave: Estudio teórico, protocolo de tratamiento, síntomas de ansiedad, terapia de grupo.

\section{Psychological group therapy to manage anxiety symptomatology}

This work present a psychological group therapy protocol to manage symptoms instead of mental disorders aimed at reducing assistance demand in Mental Health Units. The protocol consists of a group therapy program to manage anxiety symptoms from a cognitive-behavioural perspective. It includes a detailed description of the content of each session, as well as an explanatory model of anxiety and a complete checklist that considers the psychopathology and the anxiety coping strategies.
\end{abstract}

Key words: Theoretical study, treatment protocol, anxiety symptoms, group therapy.

\section{INTRODUCCIÓN}

Los trastornos mentales constituyen más del $10 \%$ del total de las enfermedades (National Institute of Mental Health, 2000) y su impacto social y económico es creciente. Concretamente, en las últimas décadas, la prevalencia e investigación en los trastornos de ansiedad ha aumentado a un ritmo mucho más rápido que en cualquier otro trastorno (Norton et al., 1995).

Según el DSM-IV-TR, dentro de los trastornos de ansiedad se pueden distinguir las siguientes categorías: trastorno de angustia, agorafobia, fobia específica, fobia social, trastorno obsesivo-compulsivo, trastorno por estrés postraumático, trastorno por estrés agudo, trastorno de ansiedad generalizada, trastorno de ansiedad debido a enfermedad médica y trastorno de ansiedad inducido por sustancias. Para cada uno de ellos se han desarrollado tratamientos específicos (Barlow et al., 2002; Botella, 2001; Clark, 1999; Echeburúa, 1998; Hahlweg et al., 2001; Stanley et al., 2003; Otto, Pollack y Maki, 2000). También existen programas polivalentes para distintos trastornos de ansiedad en población infantil y adolescente (Barret et al., 1996; Kendall et al., 1997; Lumpkin et al., 2002). Sin embargo, con población adulta, el tema está muy poco desarrollado. Algunas aproximaciones al respecto son las contribucio- 
nes de Keen (1991) y Mitchell (1999) o los programas de autoayuda mediante computador de Marks et al. (2003).

La propuesta aquí planteada incluye pacientes diagnosticados de trastorno de angustia, agorafobia, fobia específica y trastorno de ansiedad generalizada. Se excluyen el trastorno obsesivo-compulsivo, el trastorno por estrés postraumático, el trastorno por estrés agudo, la fobia social, el trastorno de ansiedad debido a enfermedades médicas y el trastorno de ansiedad inducido por sustancias debido a la idiosincrasia de su sintomatología y a la especificidad que requiere su tratamiento.

Nuestra intención es optimizar los recursos psicológicos para dar respuesta a la demanda asistencial de las Unidades de Salud Mental de la red sanitaria pública. En este sentido, nuestro protocolo tiene como objetivo disminuir la creciente presión asistencial de las Unidades de Salud Mental (mediante el tratamiento grupal) y evitar el entrenamiento reiterado del mismo contenido terapéutico en distintas patologías (tratando síntomas y no trastornos). Por tanto, la peculiaridad y originalidad de nuestra iniciativa se centra en la formulación de una estrategia terapéutica común para distintos trastornos de ansiedad que requieren la utilización de técnicas idénticas. Para este propósito se han diseñado dos sistemas de registro que permiten evaluar la intensidad de los síntomas, la adhesión al tratamiento psicológico y los resultados obtenidos con las técnicas utilizadas. Del mismo modo, se ha desarrollado un modelo explicativo que integra la psicopatología de la ansiedad y las estrategias para su afrontamiento.

\section{DESCRIPCIÓN DEL PROGRAMA DE TRATAMIENTO}

Los instrumentos de evaluación utilizados son el Inventario de Ansiedad Estado/Rasgo (STAI-E/R) de Spielberger, Gorsuch y Lushene (1970), la Escala de Adaptación (EA) de Echeburúa y Corral (1991), las Escalas de Evitación-Temor (EET) de Botella y Ballester (1997) y los auto-registros $A / B$ que han sido diseñados para este protocolo y se recogen en las Tablas 1 y 2.

Tabla 1. Auto-Registro A

Nombre:

\begin{tabular}{|l|c|c|c|c|}
\hline $\begin{array}{l}\text { Desencadenante: } \\
\text { Interno (pensamiento) } \\
\begin{array}{l}\text { Externo (situación) } \\
\text { Fecha: }\end{array}\end{array}$ & $\begin{array}{c}\text { Emoción(es) } \\
\text { (miedo, rabia inquietud, } \\
\text { tristeza, enfado, etc.) } \\
\text { Intensidad (0-10) }\end{array}$ & $\begin{array}{c}\text { Sensaciones corporales } \\
\text { (palpitaciones, ahogo, } \\
\text { sudoración, temblores) } \\
\text { Intensidad (0-10) }\end{array}$ & $\begin{array}{c}\text { Pensamiento } \\
\text { o interpretación } \\
\text { negativa / errónea } \\
\text { Intensidad (0-10) }\end{array}$ & $\begin{array}{c}\text { Conducta(s): } \\
\text { evitación y temor } \\
\text { Intensidad (0-10) }\end{array}$ \\
\hline & & & & \\
& & & & \\
\hline
\end{tabular}


Tabla 2. Auto-Registro B

Nombre:

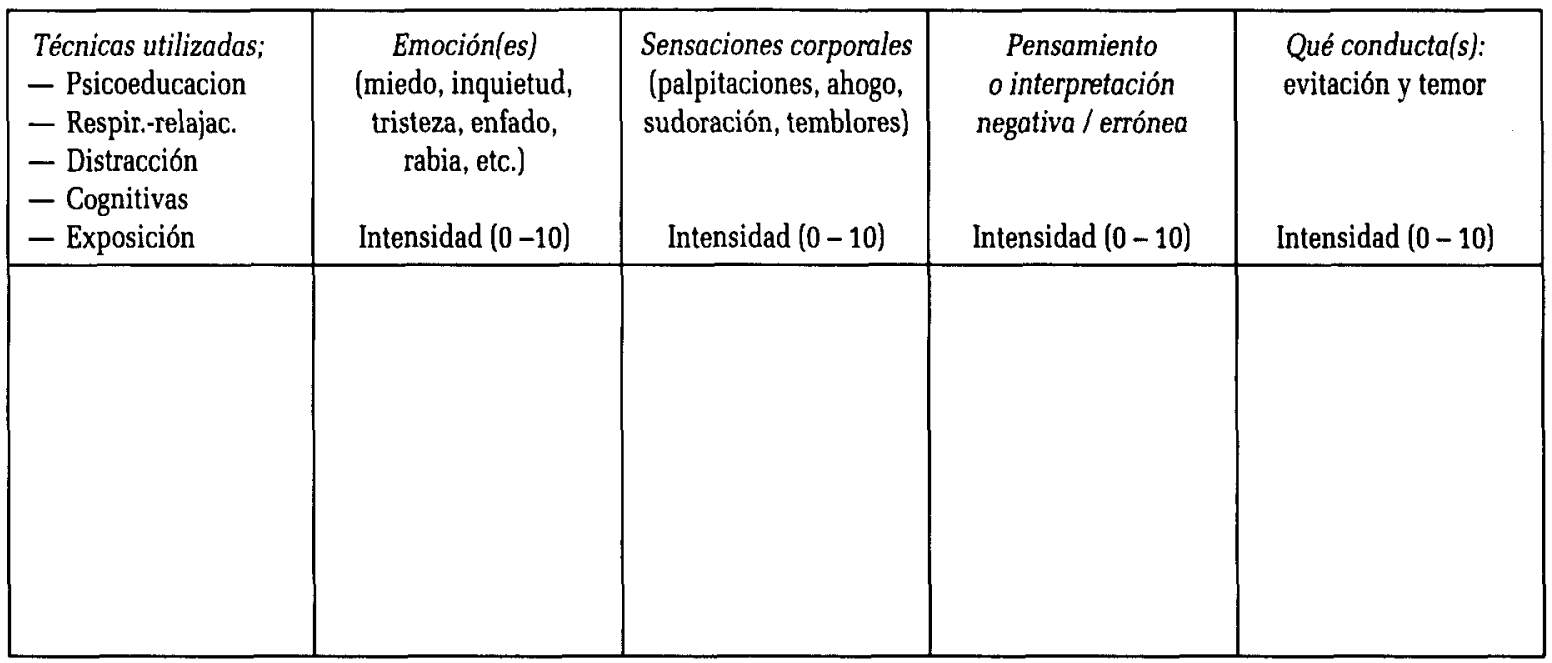

Antes de incluir a los pacientes en el programa de terapia grupal se realiza una entrevista previa en la que se determina el diagnóstico, se valora el problema, se establecen los objetivos terapéuticos y la conveniencia de participar en el tratamiento grupal.

El programa de tratamiento está estructurado en un total de 12 sesiones semanales de aproximadamente dos horas de duración cada una de ellas: una sesión de presentación del grupo y evaluación pretest, diez sesiones de tratamiento propiamente dicho y una sesión final de evaluación postest. A continuación aparece descrito el contenido de cada una de las sesiones.

\section{SESIÓN: Presentación y Pretest}

- Constituye el pretest de la evaluación. Es una sesión preliminar grupal con todos los componentes del grupo. En ella se realiza el primer pase del STAI-E/R (son dos escalas que miden la ansiedad estado y la ansiedad rasgo, respectivamente), de la EA (que evalúa el grado de interferencia del problema en distintas áreas de la vida del paciente) y de la EET (donde se evalúa el grado de evitación y temor del paciente ante distintas conductas/situaciones que teme y evita). También se familiariza a los pacientes con la utilización del auto-registro A. Además, el contenido de esta sesión se centra en la presentación de los miembros del grupo, la identificación de los pacientes en función de sus diagnósticos y en la valoración de las ventajas de la terapia grupal (p.ej., «aprender de otros", "percibir que no soy el único", "motivarme por la mejoría de otros", "comprometerme públicamente con el cambio», «beneficiarme de una asistencia pública en la que se optimizan los recursos"...).

- La tarea que se asigna a los pacientes es la cumplimentación del auto-registro A (línea-base).

\section{SESIÓN: Psicoeducación}

- Se realiza una sesión psicoeducativa cuyo objetivo es ofrecer a los pacientes información sobre distintos aspectos del concepto de ansiedad y una explicación razonable de lo que les sucede mediante la presentación de la primera parte de nuestro modelo explicativo sobre la psicopatología de la ansiedad que se ha diseñado específicamente para este protocolo (Figura 1).

- En este módulo educativo abordamos los siguientes aspectos: 


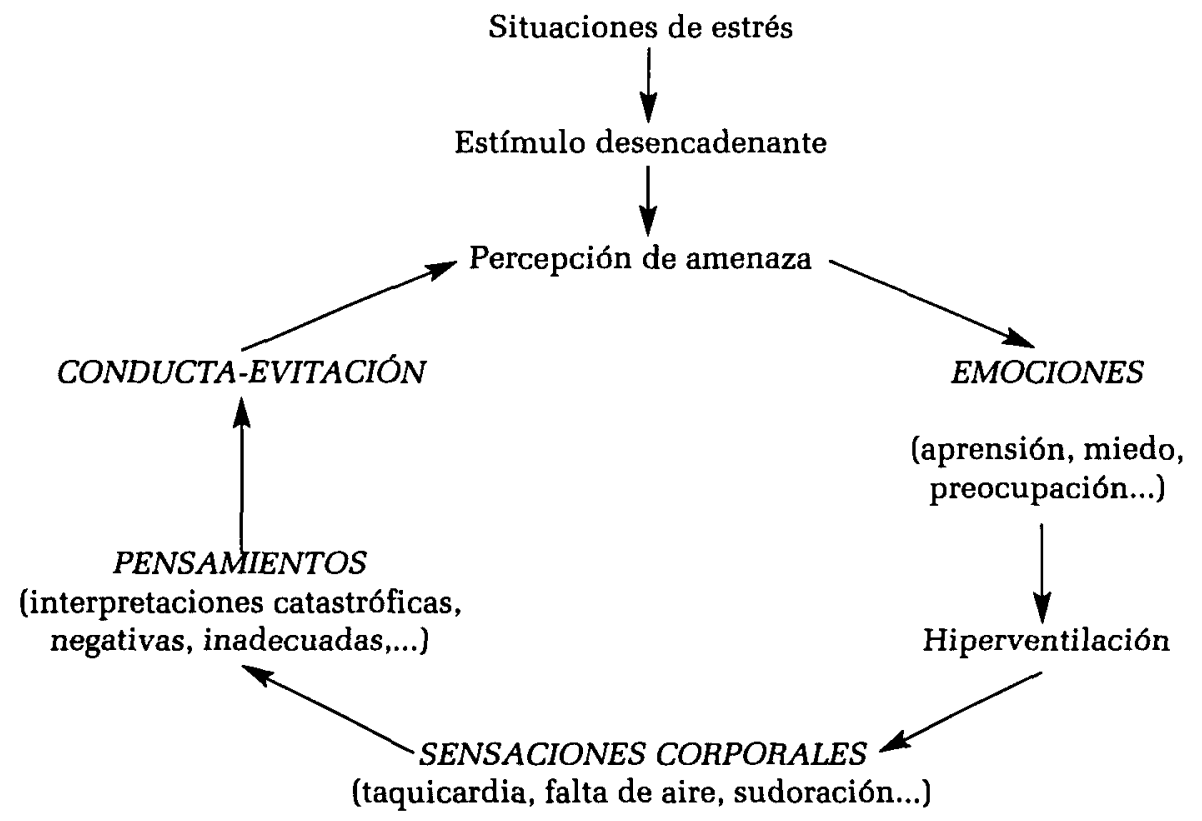

Figura 1. Modelo explicativo cognitivo-conductual I

- Qué es la ansiedad.

- Valor adaptativo / desadaptativo de la ansiedad.

- Manifestaciones de la ansiedad: fisiológica con sensaciones corporales (falta de aire, taquicardia, sudoración...); cognitiva con pensamientos ( voy a tener un ataque al corazón», "va a ocurrir algo malo», "tengo mucho temor a..."...) y conductual con comportamientos observạbles (evitar, escapar, pedir ayuda tranquilizadora, tomar medicación...).

- Introducción del modelo explicativo cognitivo-conductual I.

Toda esta información se va introduciendo a partir de ejemplos concretos de los pacientes que reflejan su sintomatología psicopatológica, en función de los distintos trastornos de ansiedad que presentan.

- La tarea que se le asigna a los pacientes es: Cumplimentar el auto-registro A, aplicar el modelo explicativo a su(s) problema(s) y hacer un resumen de la sesión.

\section{3. ${ }^{a}$ SESIÓN: Psicoeducación}

- En esta segunda sesión psicoeducativa se hace la presentación de la segunda parte de nuestro modelo explicativo que incluye las estrategias terapéuticas de afrontamiento (Figura 2).

- Mediante un estilo de intervención socrático se intenta que los pacientes vayan razonando y deduciendo cómo sería posible romper el círculo vicioso en el que se mantiene su(s) problema(s). Asimismo, se les da información general sobre las distintas estrategias de afrontamiento que podrían serles de utilidad para intentar modificar su situación actual y disminuir el malestar emocional que les genera. Dichas estrategias son: entrenamiento en respiración lenta-relajación, técnicas de distracción, reestructuración de pensamientos y ejercicios de exposición. Al igual que en la sesión anterior, el contenido se va introduciendo a partir de las aportaciones y ejemplos concretos de los pacientes.

- La tarea que se le asigna a los pacientes es: Cumplimentar el auto-registro A, siguiendo el diagrama del modelo explicativo, aplicarlo a su(s) problema(s) y hacer un resumen de la sesión.

\section{4. - 5. ${ }^{a}$ SESIÓN: Respiración-relajación}

- Estas dos sesiones tienen como objetivo abordar las sensaciones corporales mediante 


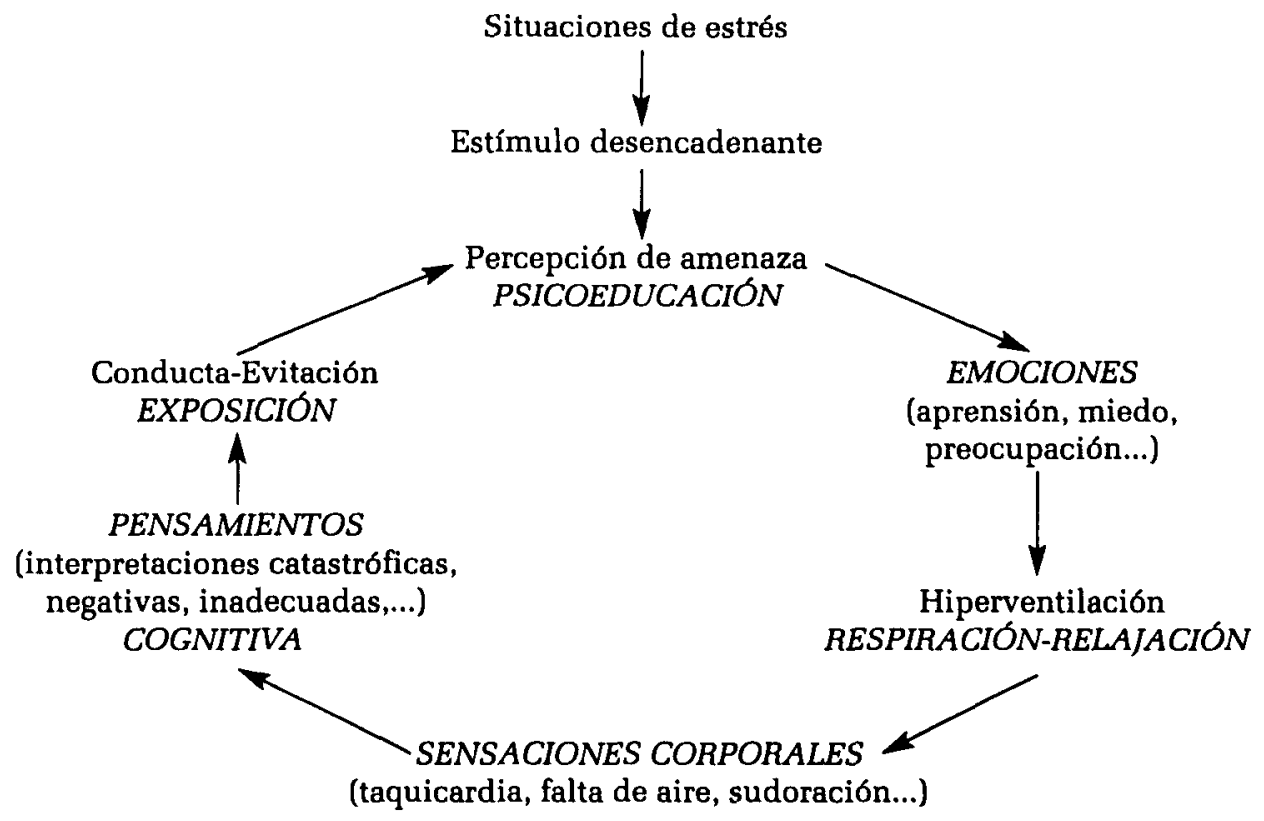

Figura 2. Modelo explicativo cognitivo-conductual II

el entrenamiento en respiración lenta-relajación. En la primera de las sesiones se comienza realizando la prueba de hiperventilación para evidenciar la importancia de la pauta de respiración en el problema y la necesidad de aprender otro ritmo de respiración. Seguidamente, se enseñan las técnicas de respiración lenta-relajación y se practican con los pacientes en posición horizontal (tumbados, sentados). En la segunda de las sesiones, se les entrena en posición vertical (de pie, andando) de forma que los pacientes aprendan a usar la técnica en condiciones más parecidas a la situación ansiógena. Los componentes que incluimos en la técnica respiración lenta relajación son:

- Respiración abdominal, cuyo objetivo es lograr una mayor oxigenación y relajación.

- Respiración asociada a relax: Pretende condicionar la relajación a un estímulo verbal repetitivo. En este caso, cada espiración se asocia con la palabra "relax».

- Autosugestión: Se trata de asociar las inspiraciones con emociones positivas (tranquilidad, calma, bienestar,...) y las espiraciones con la eliminación de emociones negativas (nerviosismo, tensión, preocupación,...).
- Distensión muscular: Consiste en trabajar la relajación de grupos musculares.

- Las tareas que se asignan a los pacientes son: cumplimentar los auto-registros $\mathrm{A}$ y B, practicar los ejercicios de respiración lentarelajación y utilizar esta estrategia terapéutica para afrontar su(s) problema(s) de ansiedad.

\section{SESIÓN: Técnicas de distracción}

- El entrenamiento en técnicas de distracción intenta ayudar a los pacientes a desviar la atención que focalizan en sus sensaciones corporales y en los pensamientos distorsionados que presentan en las situaciones de ansiedad.

- La mayoría de los pacientes han utilizado alguna estrategia de distracción para intentar combatir su(s) problema(s) de ansiedad antes de iniciar el tratamiento. Teniendo en cuenta sus propias experiencias y apoyándonos en la realización de experimentos comportamentales se les explica la importancia de la autoatención como un predisponente o una consecuencia de la ansiedad. A continuación, se concreta el papel de la autoatención en el desarrollo y manteni- 
miento del problema(s) de ansiedad de cada uno de los pacientes. Por último, se les propone aprender una serie de técnicas de distracción que pueden ayudarles a dejar de prestar atención a los estímulos amenazantes (sensaciones corporales/pensamientos). Los tipos de técnicas de distracción que se trabajan son:

- Centrar la atención en el entorno: Consiste en que los pacientes describan con detalle cualquier objeto que esté ubicado cerca de ellos teniendo en cuenta su forma, color, composición, tamaño... (p.ej., describir un cuadro, contar coches...).

- Realizar actividades mentales incompatibles: Se trata de que los pacientes desarrollen cualquier actividad mental que conlleve que centren su atención y se distraigan de su propio cuerpo y/o pensamiento (p.ej., ejercicios de cálculo, confeccionar lista de la compra, repasar la agenda de la semana...).

- Cambiar de situación y/o actividad: Se les propone a los pacientes la conveniencia de cambiar de situación o de hacer alguna actividad que les resulte suficientemente absorbente como para que dejen de centrarse en su ansiedad (p.ej., cambiar de lugar, llamar por teléfono, bañarse, hacer crucigramas...).

- La tarea que se le asigna a los pacientes es: Cumplimentar los auto-registro A y B, practicar los ejercicios de respiración lentarelajación y de distracción, y utilizar ambas estrategias terapéuticas para afrontar sus problemas de ansiedad.

\section{7. - 8. ${ }^{a}$ SESIÓN: Técnicas cognitivas}

- En estas dos sesiones se trabajan los pensamientos distorsionados mediante la discusión cognitiva, utilizando ejemplos de pensamientos negativos de cada uno de los pacientes. Los contenidos que se abordan son:

- La influencia del pensamiento en la conducta y las emociones.

- Tipos de sesgos cognitivos (sobregeneralización, error de evidencia, certeza vs. probabilidad, pensamiento todonada, pensamiento absolutista...).

- Identificación de los pensamientos negativos.

- Detención de pensamientos negativos (interrupción del curso de pensamiento ante la aparición de pensamientos negativos).

- Reestructuración de pensamientos negativos (analizar las evidencias a favor y en contra del contenido de pensamiento, probabilidades de que ocurra lo temido, interpretaciones alternativas, desdramatización o descatastrofización, y consecuencias del pensamiento negativo.

- Autoinstrucciones: monólogo interno que facilita pensamientos, emociones y conductas adaptativas.

- Las tareas asignadas a los pacientes son: cumplimentar los auto-registros A y B, identificar cada uno sus pensamientos negativos, utilizar las técnicas de detención de pensamiento, buscar explicaciones alternativas a los pensamientos negativos y registrar las autoinstrucciones empleadas.

\section{9. - 10. SESIÓN: Técnicas de exposición}

- Estas sesiones tienen como objetivo abordar la evitación mediante la técnica de exposición. Se trata de que los pacientes se expongan a los estímulos que temen sin evitarlos ni escapar de ellos. Partiendo de las Escalas de Evitación-Temor, se elabora una jerarquía de exposición y se establecen los objetivos terapéuticos para cada uno de los pacientes. Además, se les informa de la correlación positiva entre evitación y temor (a más evitación mayor miedo), de cómo el aprendizaje del miedo está asociado a determinadas situaciones, y de la manifestación del miedo y de la ansiedad a lo largo de la exposición (termómetro del miedo y curva de ansiedad).

- Las tareas asignadas a los pacientes son: cumplimentar los auto-registros A y B y llevar a cabo las tareas de exposición pautadas. 11. SESIÓN: Prevención de recaídas

- El objetivo de esta sesión consiste en la prevención de recardas. Para ello, se analiza 
la evolución del problema, atribuyendo la mejoría al tratamiento y al paciente. Además, se ofrece una visión general del contenido de las sesiones y de las técnicas aprendidas y se analizan los posibles problemas futuros y su afrontamiento.

- Las tareas asignadas a los pacientes son: cumplimentar los auto-registros A y B y seguir utilizando las técnicas adquiridas

\section{SESIÓN: Postest}

- Constituye el postest de la evaluación. En ella se realiza el pase del STAI-E/R, de la EA y de las EET. Además, se informa a los pacientes de los seguimientos establecidos.

- La tarea que se asigna a los pacientes es: cumplimentar los auto-registros A y B y seguir utilizando las técnicas adquiridas.

\section{SEGUIMIENTOS:}

- Se establecen tres momentos de seguimiento: al mes, a los 3 meses y a los 6 meses de la finalización del tratamiento. En cada una de las tres fases de seguimiento se evalúa cuál ha sido la evolución de cada paciente durante el tiempo transcurrido desde la última sesión. Además, los pacientes cumplimentan los mismos instrumentos utilizados en el pretest y postest (STAI-E/R, EA y EET).

- La tarea que se asigna a los pacientes es: cumplimentar los auto-registros A y B y seguir con la utilización de las técnicas adquiridas.

\section{DISCUSIÓN}

Actualmente, en el ámbito de la psicología clínica se observa un creciente interés por la determinación de los tratamientos empíricamente validados para síndromes clínicos específicos. Sin embargo, resulta escasa la información existente sobre la eficacia de los protocolos destinados al manejo de las manifestaciones psicopatológicas concretas que pueden darse en los distintos trastornos.
Existen estudios que han demostrado la eficacia de las distintas técnicas de tratamiento aquí expuestas (Baker et al., 2002; Barlow y Cerny, 1988; Clark y Hamsley, 1982; Clark et al., 1999; Ehlers et al., 2002; Margraft et al., 1999; Michelson y Marchione, 1991). Del mismo modo, se conocen investigaciones que evidencian resultados satisfactorios obtenidos con tratamientos grupales para trastornos específicos de ansiedad (Ballester y Botella, 1992; Dugas et al., 2003). Sin embargo, no se han encontrado estudios que hayan integrado en un mismo protocolo de evaluación y tratamiento el abordaje grupal de distintos trastornos y procedimientos terapéuticos.

Por otra parte, se tiene constancia de trabajos que han empleado un modelo explicativo de la ansiedad (Clark, 1988; 1999), pero no han incluido el componente de evitación, ni técnicas de exposición, ni se han podido aplicar indistintamente a diferentes trastornos de ansiedad como se pretende con nuestro modelo.

Asimismo, es importante señalar que, con anterioridad, se han diseñado otros sistemas de registro (Botella y Ballester, 1997), pero no han contemplado el triple sistema de respuesta de la ansiedad junto con las estrategias terapéuticas propuestas para los distintos síntomas. Con nuestro diseño, en un mismo registro se puede evaluar la intensidad de los diferentes síntomas, la adhesión al tratamiento psicológico y la eficacia obtenida con las distintas técnicas utilizadas.

Creemos que con nuestra propuesta se ofrece un tratamiento psicológico integral que recoge todos los componentes terapéuticos que han demostrado su eficacia en el tratamiento de la ansiedad. El modelo explicativo y los registros de evaluación que hemos diseñado sobre la psicopatología y tratamiento de la ansiedad 
permiten abordar la problemática de distintos trastornos con idénticos procedimientos de evaluación e intervención. En próximas publicaciones, se ofrecerán los resultados obtenidos tras la finalización del tratamiento y en cada uno de los seguimientos establecidos.

Pensamos que, dada la creciente presión asistencial existente en los Servicios de Salud Mental, se deben diseñar e implementar programas para optimizar la utilización de los recursos existentes en nuestras unidades. Tal como señala Haaga (2000), creemos que la simplificación que proporcionan los protocolos contribuye al mejor aprovechamiento de los recursos terapéuticos.

Finalmente, haciendo una generalización tal vez arriesgada de nuestra propuesta de abordar síntomas y no trastornos, cabría preguntarnos si no son básicamente similares el procedimiento terapéutico aquí expuesto y los que utilizamos para abordar la ansiedad de un psicótico, de una bulímica, de un deficiente, de un trastorno de personalidad, de un drogodependiente, o de un depresivo, por mencionar algunos trastornos. Y un dato más para la reflexión, ¿no utilizan con eficacia nuestros compañeros psiquiatras los mismos ansiolíticos para trastornos muy diversos? Esperamos que próximas investigaciones nos ayuden a desvelar interrogantes y nos permitan aproximarnos a la calidad óptima de la asistencia que deseamos.

\section{REFERENCIAS BIBLIOGRÁFICAS}

American Psychiatric Association (APA) (2002). Diagnostic and statistical manual of mental disorders (4. ${ }^{\mathrm{a}} \mathrm{Ed}$. rev.) (DSM-IV$T R$ ). Washington, DC: APA.

Baker, S.L., Patterson, M.D., y Barlow, D.H. (2002). Panic disorder and agoraphobia. In M.M. Antony and D.H. Barlow (Eds.), Handbook of assessment and treatment planning for psychological disorders (pp. 67-112). New York: Guildford Press.

Ballester, R., y Botella, C. (1992). Aplicación del programa cognitivo-comportamental de Clark y Salkovskis en grupo: Una alternativa para el tratamiento del pánico. Análisis y Modificación de Conducta, 58, 291-321.

Barlow, D.H., y Cerny, J.A. (1988). Psychological treatment of panic. New York: Guilford Press.

Barlow, D.H., Raffa, S.D., y Cohen, E.M. (2002). Psychosocial treatments for panic disorders, phobias and generalized anxiety disorder. En P.E. Nathan y J.M. Gorman (Eds.), $A$ guide to treatments that work (2." Ed.)(pp. 301-366). New York: Oxford University Press.

Barret, P.M., Duffy, A.L., Dadds, M.R., y Rapee, R.M. (1996). Family treatment of childhood anxiety: A controlled trial. Journal of Consulting and Clinical Psychology, 64, 333-342.

Botella, C. (2001). Tratamientos psicológicos eficaces para el trastorno de pánico. Psicothema, 13, 465-478.

Botella, C. y Ballester (1997). Trastorno de pánico: Evaluación y tratamiento. Barcelona: Martínez Roca.

Clark, D. (1988). A cognitive model of panic attacks. En S. Rachman y J.D. Maser (Comps.), Panic: Psychological perspectives (pp. 41-47). Nueva Jersey: Lawrence Erlbaum Associates.

Clark, D.M. (1999). Anxiety disorders: Why they persist and how to treat them. Behaviour Research and Therapy, 37, S5-S27.

Clark, D.M., y Hemsley, D.R. (1982). The effects of hyperventilation; individual variability and its relation to personality. Journal of Behavior Therapy and Experimental Psychiatry, 13, 41-47.

Clark, D.M., Salkovskis, P.M., Hackmann, A., Wells, A., Ludgate, J., y Gelder, M. (1999). Brief cognitive therapy for panic disorder: a randomized controlled trial. Journal of Consulting and Clinical Psychology, 67, 583-589.

Dugas, M.J., Ladouceur, R., Leger, E., Freeston, M.H., Langolis, F, Provencher, M.D., y Boisvert, J.M. (2003). Group cognitivebehavioral therapy for generalized anxiety disorder: Treatment outcome and long- 
term follow up. Journal of Consulting and Clinical Psychology, 71, 821-825.

Echeburúa, E. (1998). Avances en el tratamiento de los trastornos de ansiedad. Madrid: Pirámide.

Ehlers, A., Mayou, R.A., Sprigings, D., y Birkhead, J. (2000). Psychological and perceptual factors associated with arrhythmias and benign palpitations. Psychosomatic Medicine, 62, 693-702.

Haaga, D.A.F. (2000). Introduction to the special section on stepped care models in psychotherapy. Journal of Consulting and Clinical Psychology, 68, 547-548.

Hahlweg, K., Fiegenbaum, W., Frank, M., Schroeder, B., y Von Witzleben, I. (2001). Short and long-term effectiveness of an empirically supported treatment for agoraphobia. Journal of Consulting and Clinical Psychology, 69, 375-382.

Keen, J. (1991). A structured psychoeducational group program for anxiety disorders. En P.A. Keller y S.R. Heyman (Eds.), Innovations in clinical practice: $A$ source book (Vol. 10., pp. 459-468). Sarasota, FL: Professional Resource Press.

Kendall, P.C., Flanery-Schroeder, E., Panichelli-Mendel, S.M., Southam-Gerow, M., Henin, A., y Warman, M. (1997). Therapy for youths with anxiety disorders: A second randomized clinical trial. Journal of Consulting and Clinical Psychology, 65, 366-380.

Lumpkin, P.W., Silverman, W.K., Weems, C.F., Markham, M.R. y Kurtines, W.M. (2002). Treating a heterogeneous set of anxiety disorders in youths with group cognitive behavioral therapy: A partially nonconcurrent multiple baseline evaluation. Behavior Therapy, 33, 163-177.

Margraf, J., Barlow, D.H., Clark, D., y Telch, M. (1993). Psychological treatment of panic: work in progress on outcome, active ingredients and follow up. Behaviour Research and Therapy, 24, 553-567.

Marks, I.M., Mataix-Cols, D., Kenwright, M., Cameron, R., Hirsh, S., y Gega, L. (2003). Pragmatic evaluation of computer-aided self-help for anxiety and depression. British Journal of Psychiatry, 183, 57-65.

Michelson, L.K., y Marchione, K. (1991). Behavioral, cognitive, and pharmacological treatments of panic disorder with agoraphobia: Critique and synthesis. Journal of Consulting and Clinical Psychology, 59, 100-114.

Mitchell, C.G. (1999). Treating anxiety in a managed care setting: A controlled comparison of medication alone versus medication plus cognitive behavioral group therapy. Research on Social Work Practice, 9, 188-200.

National Institute of Mental Health (2000, July 6). Translating behavioral science into action: Report of the National Advisory Mental Council Behavioral Science Workgroup. Available: http://www.nimh.nih.gov/bswssummary.cfm.

Norton, G., Cox, B., Asmundson, G., y Maser, J. (1995). The growth of research on anxiety during the 1980s. Journal of Anxiety Disorders, 9, 75-85.

Otto, M.W., Pollack, M.H., y Maki, K.M. (2000). Empirically supported treatments for panic disorder: costs, benefits, and stepped care. Journal of Consulting and Clinical Psychology, 68, 556-563.

Stanley, M.A., Beck, J.G., Novy, D.M., Averill, P.M., Swann, A.C., Diefenbach, G.J., y Hopko, D.R. (2003). Cognitive-behavioral treatment of late-life generalized anxiety disorder. Journal of Consulting and Clinical Psychology, 71, 309-319. 